ISSN 1991-8631

Original Paper

http://indexmedicus.afro.who.int

\title{
Distribution spatio-temporelle des poissons Mugilidae dans la lagune de Grand-lahou (Côte d'ivoire)
}

\author{
Moustapha DIABY ${ }^{1 *}$, Konan N'DA ${ }^{2}$ et Corinne Manuella AKADJE ${ }^{2}$ \\ ${ }^{1}$ Université d'Abobo-Adjamé, Unité de Formation et de Recherches des Sciences de la Nature (UFR-SN), \\ Laboratoire de Biologie et de Cytologie Animale. 14 BP 635 Abidjan 14, Côte d'Ivoire, Tel: (225) 05344495. \\ ${ }^{2}$ Université d'Abobo-Adjamé, Unité de Formation et de Recherches des Sciences de la Nature (UFR-SN), \\ Laboratoire de Biologie et de Cytologie Animale. 02 BP 801 Abidjan 02, Côte d'Ivoire. \\ *Auteur correspondant, E-mail : diabymoustapha2002@yahoo.fr
}

\section{RESUME}

Ce travail étudie la distribution des Mugilidae dans la lagune de Grand-lahou. Des échantillons, provenant de tous les secteurs lagunaires ont été analysés de septembre 2009 à août 2010. Six espèces de Mugilidae ont été identifiées. Mugil curema (40,75\%) y domine, suivie de Liza falcipinnis $(28,96 \%)$ et de Mugil cephalus (14,96\%). Liza dumerili (7,42\%), Mugil bananensis (5,61\%) et Liza grandisquamis $(2,30 \%)$ sont peu représentées. Mugil curema et Mugil bananensis sont plus abondants au niveau de la passe et secondairement autour de ce site. Cette distribution est inversée chez Liza dumerili et Liza grandisquamis. Mugil cephalus et Liza falcipinnis ont plutôt une répartition quasi-uniforme. Les spécimens sexuellement indifférenciés sont plus distribués dans les zones proches de la passe, excepté ceux de Mugil cephalus, largement distribués dans la partie océanique. Les individus de grandes tailles restent plutôt distribués dans tous les secteurs lagunaires. De mi-juillet à fin septembre, les eaux de la lagune sont dessalées, à l'exception de celles de la partie océanique et de la passe, fréquentées par les Mugilidae pendant cette période. Cette période coïncide avec la saison de la reproduction. La sex-ratio globale est en faveur des mâles chez Liza dumerili, Mugil cephalus et Liza grandisquamis. Chez Mugil bananensis, Liza falcipinnis et Mugil curema, elle est en faveur des femelles. Les tailles moyennes varient entre les espèces. Les femelles sont significativement plus grandes que les mâles, exceptés chez Liza grandisquamis. Pour un même sexe, elles sont plus ou moins variables sur différents sites au sein de la lagune.

(C) 2012 International Formulae Group. All rights reserved.

Mots clés : Secteurs lagunaires, abondance, variabilités saisonnières, sex-ratio, structure de la population.

\section{INTRODUCTION}

Les Mugilidae sont des poissons d'origine marine, présents dans toutes les mers des régions tempérées, tropicales et subtropicales (Pombo et al., 2005). Ils affectionnent cependant, les eaux saumâtres estuariennes et lagunaires à forte variation de salinité, qu'ils remontent au cours des marées
(Albaret, 2003 ; Bernardon et Ould, 2005). Certaines espèces arrivent à supporter les conditions de vie en eau douce (Keith et Allardi, 2001). Leur présence dans les estuaires et lagunes Ouest-africains a été relevée par certains auteurs (Albaret et al., 2004 ; Harrison, 2008 ; Soyinka et al., 2010). 
En côte d'ivoire, une seule étude a été menée sur la biologie de l'écologie de ces poissons en lagune Ebrié par Albaret et Legendre (1985). Pour ces auteurs, le débarquement de ces poissons est plus important dans la lagune de Grand-lahou. Dans cette dernière lagune, bien que faisant l'objet d'une exploitation traditionnelle, leur production n'est pas mentionnée dans les statistiques de pêche. Une enquête que nous avons menée a toutefois montré un intérêt économique autour de leur exploitation. Cet intérêt est lié d'une part à l'abondance non négligeable des Mugilidae dans les débarquements et d'autre part à la qualité de la chair de ces poissons. Cependant, certaines interrogations sont régulièrement posées, notamment celles relatives à la variabilité de la richesse spécifique dans différentes zones de la lagune, ainsi que les fluctuations temporelles de leur rendement. En effet, ces poissons euryhalins, d'origine marine, soulèvent un ensemble de questions en matière d'écobiologie et de dynamique des populations. Ils sont connus comme effectuant régulièrement des migrations entre la mer et la lagune ou estuaire (Koutakis, 2004 ; Chang, 2012). Les problématiques liées à leur migration, leur pêche et à l'identification de leur stock sont donc utiles pour assurer leur gestion rationnelle. Ce travail a pour but d'étudier la distribution spatio-temporelle des espèces de Mugilidae dans la lagune de Grand-lahou, ainsi que certaines caractéristiques de leur population.

\section{MATÉRIEL ET MÉTHODES}

\section{La zone d'étude}

La lagune de Grand-lahou est située entre $5^{\circ} 07$ et $5^{\circ} 14$ de latitude nord et entre $4^{\circ}$ et $5^{\circ} 25$ de longitude ouest (Durand et Skubich, 1979). Répartie sur $190 \mathrm{~km}^{2}$ (Durand et Chantraine, 1982), elle est composée de quatre petites lagunes que sont les lagunes Tadjo, Niouzoumou, Mackey et Tagba (Lae,
1982). Les apports d'eaux continentales proviennent du fleuve Bandama et des rivières que sont le Gô et le Boubo. Sur la base des différents milieux hydrologiques au sein de la lagune (Konan et al., 2008), il a été défini quatre sites. Ce sont, le site 1 , limité à la passe et directement soumis à l'influence marine; le site 2 , très instable en termes de variation de la salinité, couvrant la zone proche de la passe et de l'embouchure du fleuve Bandama; le site 3, limité à la lagune Mackey, la partie continentale de la lagune ; le site 4 , couvrant la lagune Tadjo et la partie ouest de la lagune Niouzoumou. C'est la partie océanique de la lagune (Figure 1).

\section{Identification des espèces}

Les espèces ont été identifiées sur la base de caractères morphologiques distinctifs proposés dans la littérature, notamment chez les Mugilidae des eaux ouest-africaines (Albaret et Legendre, 1985; Diouf, 1991 ; Harrison, 2008).

\section{Répartition des espèces en lagune}

L'échantillonnage a été fait, chaque mois, sur chacun des quatre sites, de septembre 2009 à août 2010 à partir des débarquements de la pêche commerciale. Tous les spécimens débarqués le jour d'échantillonnage sont pris en compte. Sur chaque spécimen identifié, sont relevés la longueur totale au millimètre près à l'aide d'un ichtyomètre ainsi que le sexe après ouverture de la cavité abdominale.

Ont été notées: la composition spécifique (nombre d'espèces présentes); l'abondance absolue (nombre de spécimens d'une espèce dans l'échantillon); l'abondance relative (la proportion de chaque espèce dans l'échantillon). Une analyse des tableaux de la composition spécifique et des abondances a permis de comparer les sites un à un ainsi que les variations saisonnières correspondantes. 


\section{La sex-ratio}

La sex-ratio (SR), définie comme le nombre de mâles (M) pour une femelle (F), a été déterminée selon la formule $\mathrm{SR}=\mathrm{M} / \mathrm{F}$.

\section{La période de reproduction}

La migration chez certaines espèces de Mugilidae en période de reproduction a été relevée par certains auteurs (Billard, 1997 ; Fehri Bedoui et al., 2002 ; Nouiri et al., 2007). Ainsi, Chez quelques spécimens de chaque espèce, ont été notés, le poids vif, le poids des gonades, le poids éviscéré et le stade de maturité sexuelle. Le rapport gonadosomatique (RGS) mensuel a été calculé afin de situer la période de reproduction. Il s'exprime par :

RGS $=100 *$ Poids des gonades $(\mathrm{g}) /$ Poids éviscéré (g)

\section{La structure de la population}

La structure de la population a été étudiée en calculant les proportions des différentes tailles ainsi que les tailles moyennes des mâles et des femelles sur chaque site et à l'échelle de la lagune.

\section{Analyse statistique}

Les données sont analysées en utilisant un indice de la statistique descriptive à savoir le pourcentage. Le test t classique est utilisé pour vérifier la significativité des différences entre deux pourcentages. L'analyse de variance à un critère de classification (ANOVA 1) et le LSD (Least Significant Difference) de Fisher sont utilisés pour comparer les différentes tailles moyennes par site. Le Seuil de signification statistique est fixé à 5\%. Le logiciel STATISTICA Version 7.1 est utilisé à cet effet.

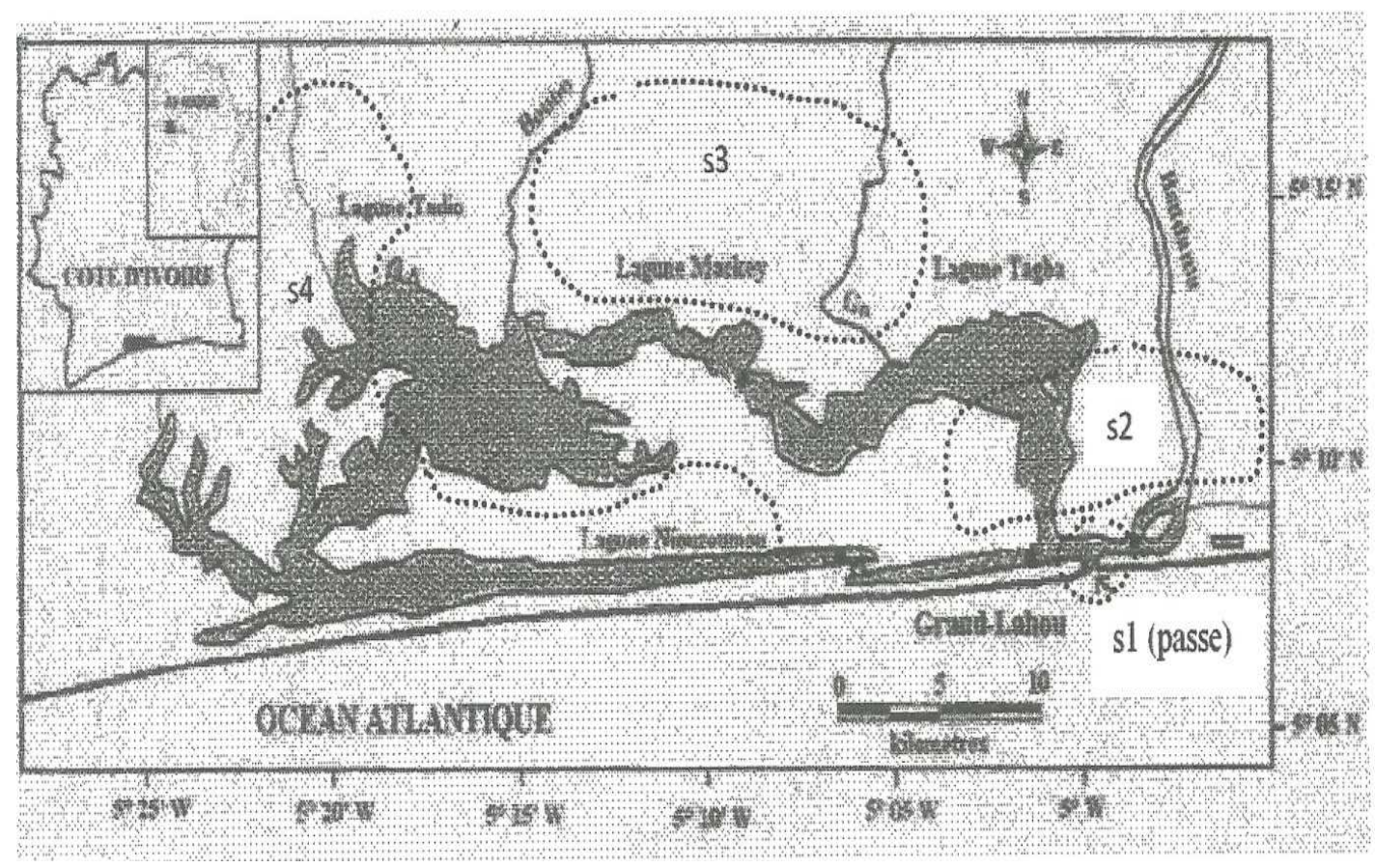

Figure 1 : Sites d'échantillonnage sur la lagune de Grand-lahou : s1, le site 1; s2, site 2; s3, site 3 ; s4, site 4 . 


\section{RESULTATS}

Abondance relative des Mugilidae en
lagune

Sur un ensemble de 8682 spécimens récoltés, Mugil curema est la plus abondante (40,75\%; $\mathrm{P}<0,001)$, suivie de Liza falcipinnis $(28,96 \%)$. Mugil cephalus arrive en troisième position. Les espèces Liza dumerili et Mugil bananensis sont faiblement représentées, encore moins Liza grandisquamis (Figure 2).

\section{Abondance relative des espèces par site}

Site 1

Mugil curema avec 59,16\% des captures y domine ( $\mathrm{P}<0,001)$, suivie de Liza falcipinnis (23,28\%). Liza dumerili, Mugil cephalus et Mugil bananensis sont peu représentées dans des proportions comparables, encore moins Liza grandisquamis (Figure 3).

Site 2

La population de Mugil curema est plus importante $(36,44 \% ; \mathrm{P}<0,001)$, suivie de celle de Liza falcipinnis (24,50\%). Mugil cephalus et Liza dumerili sont dans des proportions comparables. Liza grandisquamis et Mugil bananensis sont les moins représentées (Figure 4).

Site 3

Liza falcipinnis y est plus abondante $(53,87 \%$; $\mathrm{P}<0,001)$ suivi de Mugil cephalus $(18,82 \%)$ et de Mugil curema $(14,57 \%)$. Les autres espèces, Liza dumerili, Mugil bananensis et Liza grandisquamis, sont plus faiblement représentées (Figure 5).

Site 4

Liza falcipinnis $(39,57 \%)$ et Mugil cephalus $(39,38 \%)$ sont plus abondantes $(\mathrm{P}<$ 0,001) dans les mêmes proportions. Mugil curema arrive en troisième position dans une proportion de 9,77\%. Les espèces Liza dumerili et Mugil bananensis restent faiblement représentées dans la même proportion encore moins Liza grandisquamis (Figure 6).

\section{Périodes de reproduction}

L'évolution annuelle des valeurs du RGS présente un seul pic chez chaque espèce (Figure 7). Chez Liza dumerili et Mugil curema, ces valeurs croissent à partir de février, pour atteindre le pic en septembre, soit respectivement $18,0 \%$ et $14,7 \%$. Elles chutent de septembre à novembre. Chez Liza falcipinnis, le RGS croît à partir de novembre, pour atteindre son pic en juillet $(9,98 \%)$ et en août $(9,43 \%)$. Chez Mugil cephalus, le pic est atteint en août $(4,5 \%)$. Les valeurs chutent d'août à octobre chez ces deux espèces. Quant à Mugil bananensis, c'est à partir de septembre que le RGS croît, pour atteindre son pic en mars. Le RGS chute principalement de mai à juin.

\section{Distribution spatio-temporelle des espèces Liza dumerili}

La grande majorité de la population provient du site $2(47,67 \%$; $\mathrm{P}<0,001)$, contre $32,14 \%$ du site 1 . L'espèce reste peu présente sur les sites $4(13,20 \%)$ et $3(6,99 \%)$. De novembre à juin, l'espèce est plus présente sur le site 2 dans des proportions variant de 44 à $67 \%$, alors que cette proportion varie entre 16 et $33 \%$ sur le site 1 . Elle disparaît du site 3 de juillet à octobre et du site 2 en août et en septembre. D'août à octobre, au moins $70 \%$ de sa population est distribuée sur le site 1, coïncidant avec la période de reproduction en septembre et en octobre (Tableau 1).

\section{Liza falcipinnis}

$37,79 \%$ de la population de l'espèce proviennent du site 1 ( $\mathrm{P}<0,001)$ contre $23,35 \%$ et $24,74 \%$ respectivement des sites 2 et 4 . Seulement $14,12 \%$ de la population de l'espèce proviennent du site 3 . De novembre à juin, l'espèce reste en général régulièrement distribuée sur tous les sites, quoique présentant parfois des pics d'abondance plus ou moins remarquables sur les sites 1,2 et 3 . En août et en septembre, période de reproduction de l'espèce, sa proportion sur le site 1 est très élevée, entre 75 et $79 \%$, avant de 
chuter à 58,82\% en octobre. Comme Liza dumerili, cette espèce est absente sur les sites 2 et 3 en août et en septembre (Tableau 1).

\section{Mugil cephalus}

Cette espèce est plus distribuée sur le site 4 toute l'année que sur les autres sites dans des proportions variant de 34 à $62 \%$, et secondairement sur le site 2 de novembre à juillet entre 21 et $41 \%$ (Tableau 1). En effet, $47,73 \%$ de sa population proviennent du site 4 $(\mathrm{P}<0,001)$ contre $25,67 \%$ du site 2 . L'espèce reste peu présente sur les sites $1(17,04 \%)$ et 3 $(9,56 \%)$. En effet, elle reste faiblement représentée sur ces deux sites toute l'année. Cependant, de juillet à octobre, sa proportion devient plus importante sur le site 1 et oscille entre 28 et $52 \%$. En août, la proportion sur le site 1 est même plus élevée que sur les autres sites. La reproduction chez l'espèce intervenant après le mois d'août (Tableau 1).

\section{Mugil curema}

Les plus faibles captures sont enregistrées sur les sites $3(2,71 \%)$ et 4 $(4,35 \%)$. L'espèce reste principalement distribuée sur les sites $1 \quad(68,26 \%$ des captures ; $\mathrm{P}<0,001)$ toute l'année dans des proportions variant entre 49 et $100 \%$ (Tableau 1). $24,67 \%$ de sa population proviennent du site 2 où ses proportions mensuelles varient entre 34 et $39 \%$ de novembre à mai. Elle est absente des sites 2 et 3 en août 17,45\% et septembre. Pendant ces deux mois, toute la population de l'espèce se déplace sur le site 1 . La reproduction chez l'espèce s'étalant sur les mois de septembre et octobre (Tableau 1).

\section{Mugil bananensis}

$48,87 \%$ de la population proviennent du site $1(\mathrm{P}<0,001)$ contre $28,54 \%$ du site 2 . Sa population sur les sites $3(17,45 \%)$ et 4 $(5,13 \%)$ étant plus faible. D'octobre à juillet, l'espèce reste abondante sur les sites 1 et 2 avec des pics d'abondance partagés entre ces deux sites. La période de reproduction est centrée sur le mois de mai où sa proportion est de $55,81 \%$ sur le site 2 et $30,23 \%$ sur le site
1. Elle est absente sur les sites 2 et 3 en août et en septembre (Tableau 1).

\section{Liza grandisquamis}

Cette espèce est plus inféodée au site $2(72,00 \%$ des captures ; $\mathrm{P}<0,001)$ et secondairement au site $1(25,50 \%)$. Elle est rare sur les sites 3 et 4 dans des proportions respectives de $1,00 \%$ et $1,50 \%$. L'espèce apparait de façon spontanée entre les mois de décembre et janvier sur le site 2 (Tableau 1), alors que les femelles sont au stade de maturation sexuelle avancé.

\section{Variations spatiales de la sex-ratio}

A l'échelle de la population, les sexratios sont en faveur des mâles chez Liza dumerili, Mugil cephalus et Liza grandisquamis et sont respectivement de 1:0,88, 1:0,93 et 1:0,48. Chez Mugil bananensis, Liza falcipinnis et Mugil curema, elles sont en faveur des femelles et correspondent respectivement à 1:1,09, 1:1,07 et $1: 1,08$.

En considérant les variations de la sexratio par site, chez Liza dumerili, elle demeure en faveur des mâles sur l'ensemble des sites. Contrairement chez les espèces Liza falcipinnis et Mugil bananensis, elle est en faveur des femelles sur tous les sites. Chez Mugil curema, il existe une variation de la sex-ratio par site. Elle est en faveur des femelles sur les sites 1 et 3 et en faveur des mâles sur les sites 2 et 4 . Quant à Mugil cephalus, à l'exception du site 1 où elle est en faveur des femelles, la sex-ratio reste en faveur des mâles sur les sites 2,3 et 4 (Tableau 2).

\section{Structures de la population}

La structure de la population de Mugilidae montre que les spécimens sexuellement indifférenciés, de taille plus petite, représentent $7,86 \%$ de la population générale. En considérant les différentes espèces, cette proportion est autour de $10 \%$ chez les espèces Mugil curema, Liza dumerili 
et Mugil cephalus, encore moins chez Mugil bananensis et Liza falcipinnis dans une proportion de 3\% (Tableau 3). Ces spécimens sont principalement distribués sur le site 2 chez les espèces Mugil curema $(70,71 \%$; $\mathrm{P}<$ 0,001), Mugil bananensis (80,00\%; $\mathrm{P}<0,05)$, Liza falcipinnis $(\mathrm{P}<0,0564,47 \%$; $\mathrm{P}<0,05)$ et Liza dumerili $(77,27 \% ; \mathrm{P}<0,001)$, et secondairement sur le site 1. L'exception est notée chez Mugil cephalus, où ils sont plus distribués sur le site $4(53,50 \%$; $\mathrm{P}<0,05)$ que sur le site $2(24,20 \%)$ (Tableau 4$)$.

Les spécimens considérés comme de grande taille représentent $7,84 \%$ de la population générale. Ceux-ci sont plus distribués sur le site 1 chez Mugil curema $(88,14 \% ; \mathrm{P}<0,001)$ et Mugil bananensis $(60,32 \%)$ et secondairement sur le site 2 . Chez Liza dumerili par contre, ces gros spécimens sont représentés sur les deux sites 1 (37,19\%) et $2(43,80 \%)$ dans des proportions relevées. Enfin chez Liza falcipinnis $(49,42 \%$; $\mathrm{P}<$ $0,05)$ et Mugil cephalus $(53,85 \% ; \mathrm{P}<0,05)$, ils sont plus présents sur le site 4 et secondairement sur le site 1 chez Liza falcipinnis et sur les sites 1 et 2 chez Mugil cephalus (Tableau 4).
Quelle que soit l'espèce, la taille moyenne des femelles est significativement supérieure à celle des mâles (Test t de Student, $\mathrm{P}<0,001$ ), excepté chez Liza grandisquamis, où il n'existe pas de différence significative entre les tailles des mâles et des femelles (Test $\mathrm{t}$ de Student, $\mathrm{t}=0,99 ; \mathrm{dl}=198$; $\mathrm{P}>0,05$ ) (Tableau 5). En considérant les tailles moyennes de la population de chaque espèce par site, les plus faibles sont enregistrées sur le site 2 chez Liza dumerili, Liza falcipinnis et Mugil bananensis. L'analyse des variances suivie du test LSD de Fisher indique que ces tailles ne montrent pas de différence significative avec celles obtenues sur le site 3 et même avec celle obtenue sur le site 4 chez Mugil bananensis. Chez Mugil curema et Mugil cephalus, les tailles moyennes les plus faibles sont plutôt observées sur le site 3 . Chez Mugil cephalus, cette taille n'est pas différente de celles obtenues sur les sites 2 et 4. Les tailles moyennes les plus élevées sont observées sur le site 4 chez Liza dumerili, Liza falcipinnis et Mugil curema, sur le site $3 \mathrm{chez}$ Mugil bananensis et sur le site 1 chez Liza grandisquamis et Mugil cephalus. Ces tailles diffèrent ou non de celles obtenues sur les autres sites chez une même espèce (Tableau 5).

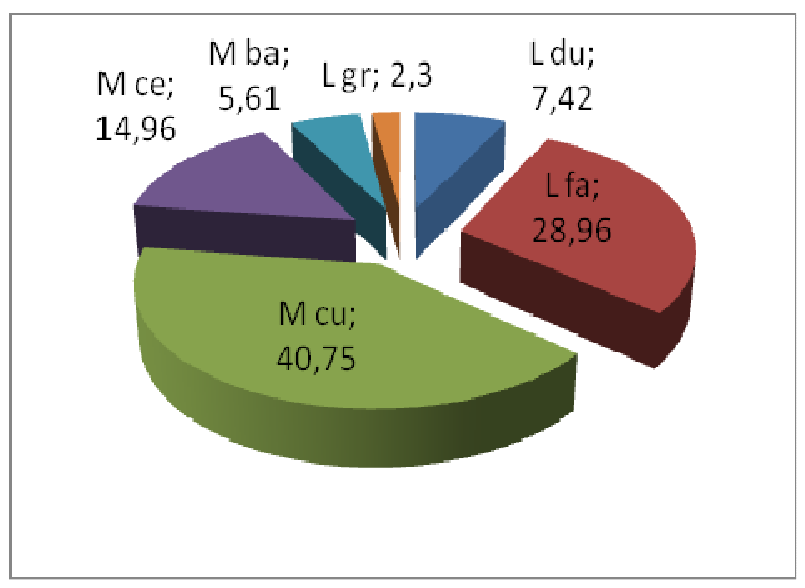

Figure 2: Proportions des espèces de Mugilidae dans la lagune de Grand-lahou. 


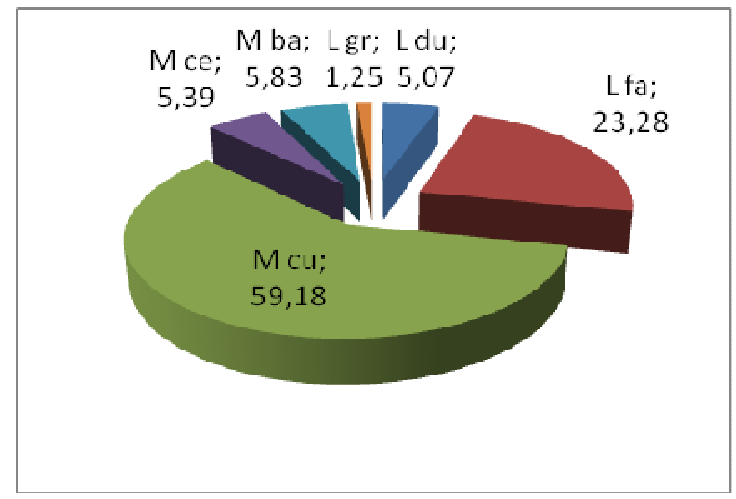

Figure 3: Proportions des espèces de Mugilidae sur le site 1.

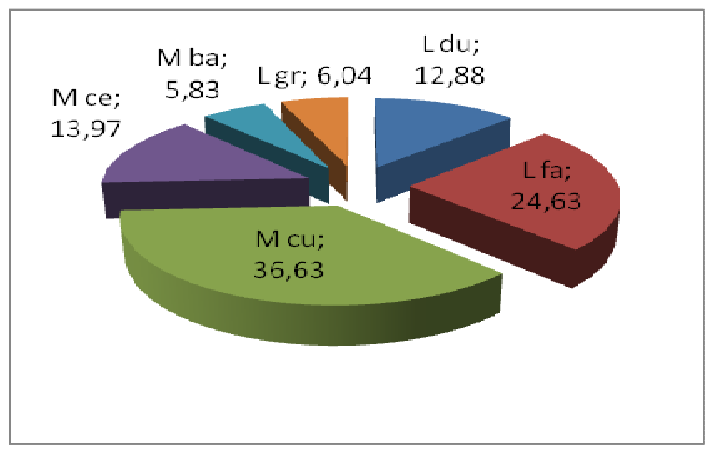

Figure 4: Proportions des espèces de Mugilidae sur le site 2.

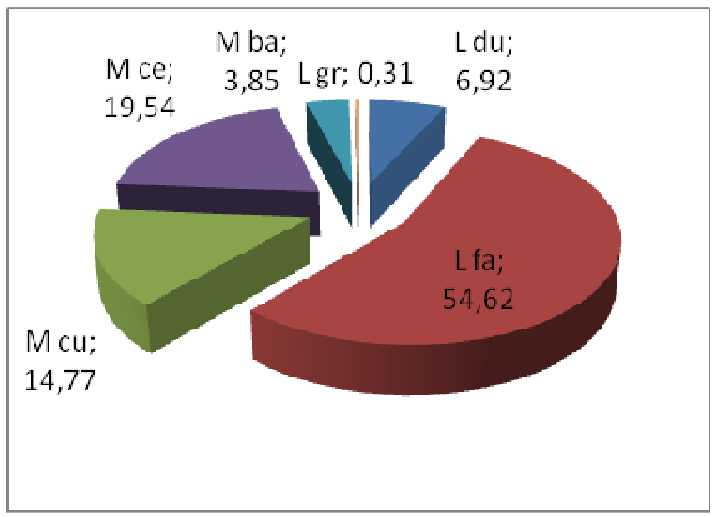

Figure 5: Proportions des espèces de Mugilidae sur le site 3. 


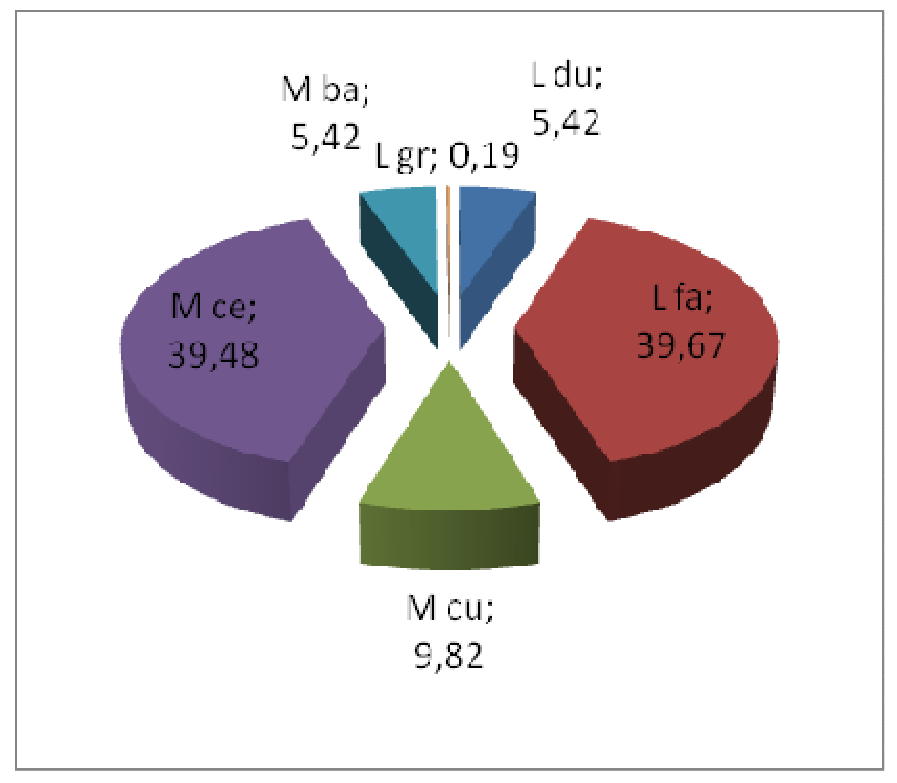

Figure 6 : Proportions des espèces de Mugilidae sur le site 4.

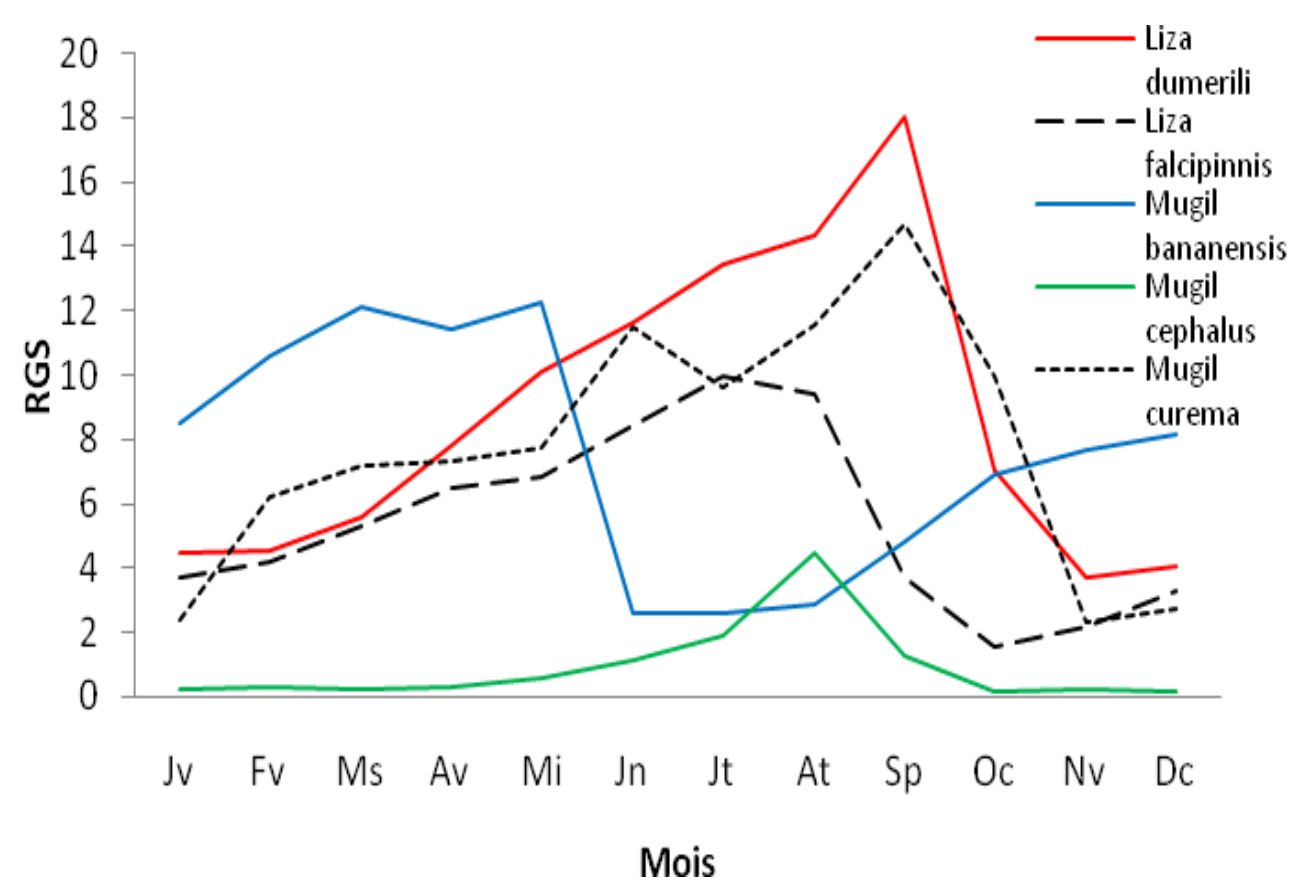

Figure 7: Evolution annuelle du rapport gonado-somatique (RGS) chez les femelles des différentes espèces de Mugilidae provenant de la lagune de Grand-Lahou. 
Tableau 1: Distribution mensuelle (\%) de l'abondance des espèces par site.

\begin{tabular}{|c|c|c|c|c|c|c|c|c|c|c|c|c|c|}
\hline Espèces & Sites & $\mathbf{J v}$ & Fv & Ms & Av & Mi & $\mathbf{J n}$ & $\mathrm{Jt}$ & At & Sp & Oc & Nv & Dc \\
\hline & site1 & 19,35 & 23,53 & 33,93 & 28,95 & 24,00 & 23,68 & 63,41 & 75,86 & 70,00 & 72,00 & 16,09 & 19,51 \\
\hline \multirow[t]{4}{*}{$L d u$} & site2 & 62,90 & 58,82 & 44,64 & 43,42 & 54,00 & 60,53 & 17,07 & 0,00 & 0,00 & 12,00 & 67,82 & 62,20 \\
\hline & site3 & 6,45 & 7,35 & 10,71 & 11,84 & 6,00 & 5,26 & 0,00 & 0,00 & 0,00 & 0,00 & 10,34 & 8,54 \\
\hline & site4 & 11,29 & 10,29 & 10,71 & 15,79 & 16,00 & 10,53 & 19,51 & 24,14 & 30,00 & 16,00 & 5,75 & 9,76 \\
\hline & site1 & 28,86 & 19,57 & 19,53 & 25,76 & 24,17 & 24,76 & 43,80 & 75,56 & 79,90 & 58,82 & 36,88 & 29,27 \\
\hline \multirow[t]{4}{*}{$L f a$} & site2 & 26,86 & 30,64 & 31,95 & 24,24 & 23,33 & 24,76 & 31,40 & 0,00 & 0,00 & 15,51 & 29,79 & 25,20 \\
\hline & site3 & 17,43 & 20,43 & 18,93 & 21,21 & 19,17 & 23,30 & 6,59 & 0,00 & 0,00 & 4,81 & 13,83 & 20,33 \\
\hline & site4 & 26,86 & 29,36 & 29,59 & 28,79 & 33,33 & 27,18 & 18,22 & 24,44 & 20,10 & 20,86 & 19,50 & 25,20 \\
\hline & site1 & 38,64 & 42,42 & 35,14 & 35,29 & 30,23 & 41,46 & 56,10 & 85,29 & 87,50 & 52,63 & 30,61 & 31,03 \\
\hline \multirow[t]{3}{*}{$M b a$} & site2 & 34,09 & 24,24 & 37,84 & 32,35 & 55,81 & 36,59 & 24,39 & 0,00 & 0,00 & 26,32 & 40,82 & 41,38 \\
\hline & site 3 & 6,82 & 12,12 & 8,11 & 5,88 & 9,30 & 7,32 & 0,00 & 0,00 & 0,00 & 0,00 & 8,16 & 6,90 \\
\hline & site4 & 20,45 & 21,21 & 18,92 & 26,47 & 4,65 & 14,63 & 19,51 & 14,71 & 12,50 & 21,05 & 20,41 & 20,69 \\
\hline & site1 & 10,87 & 7,79 & 2,67 & 4,40 & 10,31 & 6,38 & 28,05 & 52,22 & 37,70 & 42,22 & 7,91 & 10,62 \\
\hline \multirow[t]{3}{*}{ Mce } & site2 & 30,43 & 32,47 & 32,00 & 21,38 & 41,24 & 32,62 & 31,71 & 7,78 & 3,28 & 8,89 & 29,94 & 31,86 \\
\hline & site 3 & 13,04 & 12,99 & 14,67 & 11,32 & 9,28 & 9,22 & 6,10 & 0,00 & 0,00 & 1,48 & 14,69 & 18,58 \\
\hline & site4 & 45,65 & 46,75 & 50,67 & 62,89 & 39,18 & 51,77 & 34,15 & 40,00 & 59,02 & 47,41 & 47,46 & 38,94 \\
\hline \multirow{4}{*}{ M сu } & site1 & 51,69 & 50,21 & 49,65 & 51,61 & 51,01 & 66,35 & 69,69 & 98,06 & 100,00 & 77,71 & 50,77 & 50,17 \\
\hline & site2 & 39,70 & 35,27 & 39,72 & 37,63 & 34,23 & 27,96 & 26,55 & 0,00 & 0,00 & 17,20 & 35,38 & 38,21 \\
\hline & site3 & 2,25 & 4,98 & 5,67 & 5,38 & 5,37 & 1,66 & 0,66 & 0,00 & 0,00 & 0,00 & 7,18 & 6,64 \\
\hline & site 4 & 6,37 & 9,54 & 4,96 & 5,38 & 9,40 & 4,03 & 3,10 & 1,94 & 0,00 & 5,10 & 6,67 & 4,98 \\
\hline \multirow{4}{*}{$L g r$} & site1 & 7,35 & 13,16 & 42,86 & 83,33 & 66,67 & 50,00 & 66,67 & 100,00 & 100,00 & 37,50 & 60,00 & 21,95 \\
\hline & site2 & 91,18 & 86,84 & 42,86 & 16,67 & 16,67 & 50,00 & 33,33 & 0,00 & 0,00 & 62,50 & 20,00 & 78,05 \\
\hline & site3 & 1,47 & 0,00 & 0,00 & 0,00 & 0,00 & 0,00 & 0,00 & 0,00 & 0,00 & 0,00 & 10,00 & 0,00 \\
\hline & site4 & 0,00 & 0,00 & 14,29 & 0,00 & 16,67 & 0,00 & 0,00 & 0,00 & 0,00 & 0,00 & 10,00 & 0,00 \\
\hline
\end{tabular}

Tableau 2: Variations spatiales de la sex-ratio dans la population des Mugilidae de la lagune de Grand-Lahou.

\begin{tabular}{|c|c|c|c|c|c|c|c|c|c|c|c|}
\hline & & $n$ & M & F & $\begin{array}{l}\text { S / R } \\
M: F\end{array}$ & & & $\mathrm{n}$ & $\mathbf{M}$ & F & $\begin{array}{l}\text { S / R } \\
M \cdot F\end{array}$ \\
\hline \multirow{5}{*}{$L d u$} & Site 1 & 197 & 101 & 96 & $1: 0,95$ & \multirow{5}{*}{ Mce } & Site 1 & 203 & 98 & 105 & $1: 1,07$ \\
\hline & Site 2 & 256 & 135 & 121 & $1: 0,90$ & & Site 2 & 295 & 153 & 142 & $1: 0,93$ \\
\hline & Site 3 & 42 & 29 & 13 & $1: 0,45$ & & Site 3 & 109 & 62 & 47 & $1: 0,76$ \\
\hline & Site 4 & 83 & 43 & 40 & $1: 0,93$ & & Site 4 & 535 & 279 & 256 & $1: 0,92$ \\
\hline & Lagune & 578 & 308 & 270 & $1: 0,88$ & & Lagune & 1142 & 592 & 550 & $1: 0,93$ \\
\hline \multirow{5}{*}{$L f a$} & Site 1 & 933 & 457 & 476 & $1: 1,04$ & \multirow{5}{*}{$M b a$} & Site 1 & 235 & 109 & 126 & $1: 1,16$ \\
\hline & Site 2 & 538 & 249 & 289 & $1: 1,16$ & & Site 2 & 127 & 63 & 64 & $1: 1,02$ \\
\hline & Site 3 & 346 & 166 & 180 & $1: 1,08$ & & Site 3 & 25 & 12 & 13 & $1: 1,08$ \\
\hline & Site 4 & 621 & 308 & 313 & $1: 1,02$ & & Site 4 & 85 & 40 & 45 & $1: 1,13$ \\
\hline & Lagune & 2438 & 1180 & 1258 & $1: 1,07$ & & Lagune & 472 & 224 & 248 & $1: 1,11$ \\
\hline
\end{tabular}




\begin{tabular}{|c|c|c|c|c|c|c|c|c|c|c|c|}
\hline \multirow{5}{*}{$M c u$} & Site 1 & 2339 & 1099 & 1240 & $1: 1,13$ & \multirow{5}{*}{$L g r$} & Site 1 & 51 & 40 & 11 & $1: 0,28$ \\
\hline & Site 2 & 615 & 312 & 303 & $1: 0,97$ & & Site 2 & 144 & 90 & 54 & $1: 0,60$ \\
\hline & Site 3 & 63 & 30 & 33 & $1: 1,10$ & & Site 3 & 2 & 2 & 0 & - \\
\hline & Site 4 & 153 & 77 & 76 & $1: 0,99$ & & Site 4 & 3 & 3 & 0 & - \\
\hline & Lagune & 3170 & 1518 & 1652 & $1: 1,09$ & & Lagune & 200 & 135 & 65 & $1: 0,48$ \\
\hline
\end{tabular}

Tableau 3: Proportions des spécimens sexuellement indifférenciés (I) et des spécimens de grande taille (GS) dans la population de chaque espèce. GS, tailles $>60 \mathrm{~cm}$ chez Mugil cephalus; tailles > $30 \mathrm{~cm}$ chez les autres espèces.

\begin{tabular}{ccccccc}
\hline & $\begin{array}{c}\text { Liza } \\
\text { dumerili } \\
\text { Effectif (\%) }\end{array}$ & $\begin{array}{c}\text { Mugil } \\
\text { bananensis } \\
\text { Effectif (\%) }\end{array}$ & $\begin{array}{c}\text { Mugil } \\
\text { curema } \\
\text { Effectif (\%) }\end{array}$ & $\begin{array}{c}\text { Liza } \\
\text { falcipinnis } \\
\text { Effectif (\%) }\end{array}$ & $\begin{array}{c}\text { Mugil } \\
\text { cephalus } \\
\text { Effectif }(\%)\end{array}$ & $\begin{array}{c}\text { Liza } \\
\text { grandisquamis } \\
\text { Effectif (\%) }\end{array}$ \\
\hline I & $66(10,25)$ & $15(3,08)$ & $368(10,40)$ & $76(3,02)$ & $157(12,09)$ & 0 \\
GS & $121(18,79)$ & $63(12,94)$ & $177(5,00)$ & $242(9,63)$ & $78(6,00)$ & 0 \\
Total & 644 & 487 & 3538 & 2514 & 1299 & 200 \\
\hline
\end{tabular}

Tableau 4: Proportions des spécimens sexuellement indifférenciés (I) et des spécimens de grande taille (GS) par site. GS, tailles $>60 \mathrm{~cm}$ chez Mugil cephalus; tailles $>30 \mathrm{~cm}$ chez les autres espèces.

\begin{tabular}{lcccccc}
\hline Espèces & & Site 1 & Site 2 & Site 3 & Site 4 & Effectif \\
\hline \multirow{2}{*}{ Liza dumerili } & I & $10(15,15)$ & $51(77,27)$ & $3(4,55)$ & $2(3,03)$ & 66 \\
& GS & $45(37,19)$ & $53(43,80)$ & $7(5,79)$ & $16(13,22)$ & 121 \\
\hline \multirow{2}{*}{ Liza falcipinnis } & I & $17(22,37)$ & $49(64,47)$ & $9(11,84)$ & $1(1,32)$ & 15 \\
& GS & $38(60,32)$ & $13(20,63)$ & $5(7,94)$ & $7(11,11)$ & 63 \\
\hline \multirow{2}{*}{ Mugil curema } & I & $76(20,65)$ & $258(70,11)$ & $33(8,97)$ & $1(0,27)$ & 368 \\
& GS & $156(88,14)$ & $10(5,65)$ & $1(0,56)$ & $10(5,65)$ & 177 \\
\hline \multirow{2}{*}{ Mugil cephalus } & $\mathrm{I}$ & $17(10,83)$ & $38(24,20)$ & $18(11,46)$ & $84(53,50)$ & 76 \\
& GS & $112(32,75)$ & $45(13,16)$ & $16(4,68)$ & $169(49,42)$ & 242 \\
\hline \multirow{2}{*}{ Mugil bananensis } & $\mathrm{I}$ & $3(20)$ & $12(80)$ & 0 & 0 & 157 \\
& $\mathrm{GS}$ & $15(19,23)$ & $18(23,08)$ & $3(3,85)$ & $42(53,85)$ & 78 \\
\hline \multirow{2}{*}{ Liza grandisquamis } & $\mathrm{I}$ & 0 & 0 & 0 & 0 & 0 \\
& $\mathrm{GS}$ & 0 & 0 & 0 & 0 & 0 \\
\hline
\end{tabular}


Tableau 5: Tailles moyennes spécifiques des mâles, des femelles et des spécimens sexuellement indifférenciés (I).

\begin{tabular}{|c|c|c|c|c|c|c|c|c|c|c|}
\hline \multirow[b]{2}{*}{ Espèces } & \multicolumn{3}{|c|}{ Femelles } & \multicolumn{2}{|c|}{ Mâles } & \multicolumn{5}{|c|}{ Population } \\
\hline & & $\mathbf{N}$ & Moy & $\mathbf{N}$ & Moy & NS & & $\mathbf{N}$ & Moy & NS \\
\hline \multirow{6}{*}{ Mce } & S 1 & 105 & $54,60^{ \pm} 7,10$ & 98 & $48,29^{ \pm} 5,62$ & $(\mathrm{~F} / \mathrm{M})^{*}$ & PS 1 & 220 & $50,14^{ \pm} 8,49$ & $(\mathrm{PS} 1 / \mathrm{PS} 2)^{*}$ \\
\hline & S 2 & 142 & $53,79^{ \pm} 5,32$ & 153 & $48,09^{ \pm} 4,66$ & $(\mathrm{~F} / \mathrm{M})^{*}$ & PS 2 & 333 & $48,60^{ \pm} 8,28$ & $(\mathrm{PS} 1 / \mathrm{PS} 3)^{*}$ \\
\hline & S 3 & 47 & $53,61^{ \pm} 4,83$ & 62 & $47,94^{ \pm} 4,30$ & $(\mathrm{~F} / \mathrm{M})^{*}$ & PS 3 & 127 & $47,67^{ \pm} 8,35$ & $(\mathrm{PS} 1 / \mathrm{PS} 4)^{*}$ \\
\hline & S 4 & 256 & $54,39^{ \pm} 5,35$ & 279 & $46,89^{ \pm} 4,95$ & $(\mathrm{~F} / \mathrm{M})^{*}$ & PS 4 & 619 & $48,04^{ \pm} 8,60$ & PS2 / PS3 \\
\hline & & & & & & & I & 157 & $32,13^{ \pm} 2,70$ & PS2/ PS4 \\
\hline & $\mathrm{P}$ & 550 & $54,21^{ \pm} 5,67$ & 592 & $47,54^{ \pm} 4,96$ & $(\mathrm{~F} / \mathrm{M})^{*}$ & $\mathrm{P}$ & 1299 & $48,50^{ \pm} 8,51$ & PS3 / PS4 \\
\hline \multirow{6}{*}{$L d u$} & S 1 & 96 & $29,70^{ \pm} 5,22$ & 101 & $21,10^{ \pm} 3,43$ & $(\mathrm{~F} / \mathrm{M})^{*}$ & PS 1 & 207 & $24,79^{ \pm} 6,40$ & $(\mathrm{PS} 1 / \mathrm{PS} 2)^{*}$ \\
\hline & S 2 & 121 & $28,84^{ \pm} 5,02$ & 135 & $21,47^{ \pm} 2,86$ & $(\mathrm{~F} / \mathrm{M})^{*}$ & PS 2 & 307 & $23,23^{ \pm} 6,31$ & PS1 / PS3 \\
\hline & S 3 & 13 & $29,47^{ \pm} 4,53$ & 29 & $22,88^{ \pm} 2,83$ & $(\mathrm{~F} / \mathrm{M})^{*}$ & PS 3 & 45 & $24,21^{ \pm} 5,17$ & PS1 / PS4 \\
\hline & S 4 & 40 & $28,82^{ \pm} 5,09$ & 43 & $22,22^{ \pm} 3,63$ & $(\mathrm{~F} / \mathrm{M})^{*}$ & PS 4 & 85 & $25,15^{ \pm} 5,65$ & PS2 / PS3 \\
\hline & & & & & & & I & 66 & $14,63^{ \pm} 0,47$ & (PS2/ PS4)* \\
\hline & $\mathrm{P}$ & 270 & $29,18^{ \pm} 5,07$ & 308 & $21,58^{ \pm} 3,20$ & $(\mathrm{~F} / \mathrm{M}) *$ & $\mathrm{P}$ & 644 & $24,05^{ \pm} 6,22$ & PS3 / PS4 \\
\hline \multirow{6}{*}{$L f a$} & S 1 & 308 & $25,83^{ \pm} 4,14$ & 308 & $22,68^{ \pm} 2,59$ & $(\mathrm{~F} / \mathrm{M})^{*}$ & PS 1 & 950 & $24,78^{ \pm} 4,20$ & $(\mathrm{PS} 1 / \mathrm{PS} 2)^{*}$ \\
\hline & S 2 & 289 & $25,17^{ \pm} 4,28$ & 249 & $21,82^{ \pm} 2,57$ & $(\mathrm{~F} / \mathrm{M})^{*}$ & PS 2 & 587 & $23,00^{ \pm} 4,32$ & (PS1 / PS3)* \\
\hline & S 3 & 180 & $24,43^{ \pm} 3,59$ & 166 & $21,93^{ \pm} 2,37$ & $(\mathrm{~F} / \mathrm{M})^{*}$ & PS 3 & 355 & $23,07^{ \pm} 3,41$ & $(\mathrm{PS} 1 / \mathrm{PS} 4)^{*}$ \\
\hline & S 4 & 313 & $29,91^{ \pm} 3,92$ & 308 & $24,76^{ \pm} 3,17$ & $(\mathrm{~F} / \mathrm{M})^{*}$ & PS 4 & 622 & $27,34^{ \pm} 4,41$ & PS2 / PS3 \\
\hline & & & & & & & I & 76 & $16,48^{ \pm} 1,15$ & (PS2/ PS4)* \\
\hline & $\mathrm{P}$ & 1258 & $26,83^{ \pm} 4,59$ & 1180 & $23,08^{ \pm} 2,98$ & $(\mathrm{~F} / \mathrm{M})^{*}$ & $\mathrm{P}$ & 2514 & $24,76^{ \pm} 4,50$ & $(\mathrm{PS} 3 / \mathrm{PS} 4)^{*}$ \\
\hline \multirow{6}{*}{$M c u$} & S 1 & 1240 & $25,70^{ \pm} 3,71$ & 1099 & $22,26^{ \pm} 2,09$ & $(\mathrm{~F} / \mathrm{M})^{*}$ & PS 1 & 2415 & $23,85^{ \pm} 3,70$ & $(\mathrm{PS} 1 / \mathrm{PS} 2)^{*}$ \\
\hline & S 2 & 303 & $24,45^{ \pm} 2,84$ & 312 & $21,84^{ \pm} 2,21$ & $(\mathrm{~F} / \mathrm{M})^{*}$ & PS 2 & 873 & $21,06^{ \pm} 4,04$ & (PS1 / PS3)* \\
\hline & S 3 & 33 & $22,93^{ \pm} 2,62$ & 30 & $20,89^{ \pm} 1,71$ & $(\mathrm{~F} / \mathrm{M})^{*}$ & PS 3 & 96 & $20,02^{ \pm} 3,40$ & $(\mathrm{PS} 1 / \mathrm{PS} 4)^{*}$ \\
\hline & S 4 & 76 & $26,66^{ \pm} 2,82$ & 77 & $22,73^{ \pm} 2,30$ & $(\mathrm{~F} / \mathrm{M})^{*}$ & PS 4 & 154 & $24,63^{ \pm} 3,27$ & (PS2 / PS3)* \\
\hline & & & & & & & I & 368 & $16,24^{ \pm} 1,10$ & (PS2/ PS4)* \\
\hline & $\mathrm{P}$ & 1652 & $25,46^{ \pm} 3,57$ & 1518 & $22,17^{ \pm} 2,14$ & $(\mathrm{~F} / \mathrm{M})^{*}$ & $\mathrm{P}$ & 3538 & $23,09^{ \pm} 3,99$ & $(\mathrm{PS} 3 / \mathrm{PS} 4)^{*}$ \\
\hline \multirow{6}{*}{$M b a$} & S 1 & 126 & $27,67^{ \pm} 3,34$ & 109 & $22,86^{ \pm} 2,80$ & $(\mathrm{~F} / \mathrm{M})^{*}$ & PS 1 & 238 & $25,32^{ \pm} 4,04$ & (PS1 / PS2) \\
\hline & S 2 & 64 & $27,64^{ \pm} 2,92$ & 63 & $22,90^{ \pm} 2,45$ & $(\mathrm{~F} / \mathrm{M})^{*}$ & PS 2 & 139 & $24,43^{ \pm} 4,44$ & PS1 / PS3 \\
\hline & S 3 & 13 & $28,25^{ \pm} 4,31$ & 12 & $22,78^{ \pm} 1,97$ & $(\mathrm{~F} / \mathrm{M})^{*}$ & PS 3 & 25 & $25,62^{ \pm} 4,34$ & PS1 / PS4 \\
\hline & S 4 & 45 & $26,81^{ \pm} 3,27$ & 40 & $21,91^{ \pm} 1,79$ & $(\mathrm{~F} / \mathrm{M})^{*}$ & PS 4 & 85 & $24,50^{ \pm} 3,63$ & PS2 / PS3 \\
\hline & & & & & & & I & 15 & $15,45^{ \pm} 1,13$ & PS2/ PS4 \\
\hline & $\mathrm{P}$ & 248 & $27,54^{ \pm} 3,28$ & 224 & $22,70^{ \pm} 2,52$ & $(\mathrm{~F} / \mathrm{M}) *$ & $\mathrm{P}$ & 487 & $24,94^{ \pm} 4,12$ & $\mathrm{PS} 3$ / PS4 \\
\hline \multirow{6}{*}{$L g r$} & S 1 & 11 & $21,88^{ \pm} 1,93$ & 40 & $21,25^{ \pm} 3,35$ & $\mathrm{~F} / \mathrm{M}$ & PS 1 & 51 & $21,39^{ \pm} 3,09$ & $(\mathrm{PS} 1 / \mathrm{PS} 2)^{*}$ \\
\hline & S 2 & 54 & $20,46^{ \pm} 1,64$ & 90 & $19,98^{ \pm} 2,37$ & $\mathrm{~F} / \mathrm{M}$ & PS 2 & 144 & $20,16^{ \pm} 2,13$ & PS1 / PS3 \\
\hline & S 3 & 0 & & 2 & & & PS 3 & 2 & & \\
\hline & S 4 & 0 & & 3 & & & PS 4 & 3 & & \\
\hline & & & & & & & I & 0 & & \\
\hline & $\mathrm{P}$ & 65 & $20,70^{ \pm} 1,76$ & 135 & $20,33^{ \pm} 2,73$ & $\mathrm{~F} / \mathrm{M}$ & $\mathrm{P}$ & 200 & $20,45^{ \pm} 2,46$ & PS3 / PS4 \\
\hline
\end{tabular}




\section{DISCUSSION}

Six espèces de Mugilidae ont été identifiées dans la lagune de Grand-lahou. Ce sont, Mugil curema, Liza falcipinnis, Mugil cephalus, Liza dumerili, Mugil bananensis et Liza grandisquamis. Cinq de ces six espèces ont été identifiées dans la lagune Ebrié, contigüe à celle de Grand-lahou, exceptée l'espèce Mugil bananensis, plutôt signalée le long du littoral en milieu marin (Albaret et Legendre, 1985). Cette lagune voisine présente des milieux hydrologiques similaires à celle de Grand-lahou, à savoir des secteurs estuarien et continental (Durand et Chantraine, 1982). Cependant, ces six espèces sont régulièrement rencontrées dans les lagunes ou estuaires ouest-africains quoique une septième espèce, Liza bandialensis, ait été localisée, dans des estuaires à Sine Saloum (Diouf, 1991 ; Harrison, 2008 ; Trape et al., 2009).

Ces différentes espèces colonisent les différents milieux hydrologiques au sein de la lagune. Ceci dénote de leur caractère euryhalin (Keith et Allardi, 2001; Harrison, 2008). Cependant, une analyse des abondances de chaque espèce par site, met en évidence des spécificités dans leur occupation spatiale. En effet, Mugil curema et Mugil bananensis, sont principalement inféodées à la passe (site 1) et secondairement dans les zones plus proches de la passe (site 2). Cette distribution est plutôt inversée chez Liza dumerili et Liza grandisquamis. L'influence marine étant plus prononcée à la passe que sur le site 2 (Konan et al., 2008). Enfin, Liza falcipinnis et Mugil cephalus sont réparties quasi uniformément sur toute la lagune. Albaret et Legendre (1985) ont mené une étude similaire chez les Mugilidae de la lagune Ebrié, lagune contigüe à la lagune de Grand-lahou. Ils ont aussi relevé, la forte abondance de Mugil curema et de Liza grandisquamis au niveau de la passe, zone sous forte influence marine. Cependant, ils notent le pic d'abondance chez Liza grandisquamis au niveau de la passe que dans les alentours de la zone, contrairement aux observations actuelles. Ils signalent enfin la répartition uniforme de Liza falcipinnis sur toute la lagune, conformément aux présents résultats.

Par ailleurs, il a été mis en évidence le rôle de la salinité sur la distribution chez les Mugilidae (Cardona, 2000 ; Cardona, 2006). Toutefois, Il a été signalé l'absence de ces Mugilidae sur les sites 2 et 3 de mi-juillet à fin septembre, la période post-saison des pluies où la crue des cours d'eau continentaux se déversant dans la lagune entraîne sa dessalure (Konan et al., 2008,). Leur présence en lagune est donc limitée aux sites 1 et 4 . Cette absence sur les sites 2 et 3 serait liée à la préférence des eaux saumâtres par rapport à l'eau douce, les Mugilidae étant des poissons euryhalins d'origine marine, qui affectionnent particulièrement les eaux saumâtres (Albaret, 2003).

L'analyse de l'évolution du RGS montre que la période des pluies coïncide avec la saison de reproduction chez Mugil curema, Liza falcipinnis, Mugil cephalus et Liza dumerili. Il pourrait s'agir aussi d'une migration vers les lieux de reproduction. En effet, des auteurs relèvent une migration chez les Mugilidae en période de reproduction vers la mer (Ameur, 2003; Perez-Ruzafa et al., 2004). Cependant, des cas de reproduction en eau saumâtre ou douce sont rapportés (ElZarka et El-Sedfy, 1970; Bruslé, 1981). Parmi les espèces soumises à l'étude, il a été noté que Mugil cephalus se reproduit en mer (Ould Mohamed Vall, 2004) de même que Mugil curema (Marin et al., 2003). La reproduction chez Liza falcipinnis et Liza grandisquamis a été signalée dans la lagune Ebrié et des spécimens de Liza dumerili et Mugil curema ont été trouvés à un stade de 
maturation très avancé (Albaret et Legendre, 1985 ; Albaret, 1994). Nos travaux en cours ont identifié des femelles aux stades de maturité sexuelle avancée et de stade post ponte principalement sur le site 1 et rarement sur le site 4, ces deux sites étant les seuls fréquentés pendant cette période.

La sex-ratio est en faveur des mâles chez les espèces, Mugil cephalus, Liza dumerili, et Liza grandisquamis. Elle l'est plutôt chez les femelles chez Mugil curema, Liza falcipinnis et Mugil bananensis. Plusieurs hypothèses peuvent justifier la prédominance des mâles ou des femelles, entre autres la sélectivité des engins de pêche (Chikou, 2006). Notre étude a montré une prédominance des mâles ou des femelles chez chacune des espèces étudiées selon les classes de tailles, traduisant une évolution de la sexratio en fonction de la taille de capture. Ainsi, les classes de tailles les plus accessibles aux engins de pêche ont une influence directe sur la sex-ratio de la population. A cet effet, Albaret et Legendre (1985) ont signifié avoir utilisé un seul type d'engin peu sélectif au cours de leur échantillonnage en lagune Ebrié, contrairement à notre étude, où les engins utilisés sont les éperviers et surtout les filets maillants de mailles variant de 20 à $50 \mathrm{~mm}$, ces engins étant très sélectifs (Deschamps, 2009). Ce qui pourrait expliquer les différences de nos résultats. De plus, Plisnier cité par chikou (2006) note que des poissons peuvent présenter un comportement grégaire fortement lié au sexe. Les Mugilidae sont connus comme étant des poissons grégaires chez lesquels il existe une ségrégation par classe d'âge et par sexe au cours de leur déplacement (Ould Mohamed Vall, 2004). Ainsi, les bancs les plus accessibles aux engins de pêche ont une influence directe sur la sex-ratio. La pêche aux mêmes endroits a tendance à prélever les individus d'un sexe plus qu'un autre. Lors des présentes investigations, il a été constaté que les pêcheurs avaient l'habitude de pêcher dans les mêmes secteurs. Quant aux variations de la sex-ratio par site constatées chez certaines espèces, elles peuvent être liées à une ségrégation selon le sexe, une variation dans l'occupation spatiale de chaque banc, une répartition différentielle selon la taille et l'âge, une mortalité naturelle sélective (Bruslé et Bruslé, 1977). Cependant, cette étude ne permet pas de vérifier ces hypothèses.

Les spécimens sexuellement indifférenciés sont préférentiellement distribués sur le site 2, à l'exception de Mugil cephalus, chez lequel ils sont plus présents sur le site 4 et secondairement sur le site 2 . Chez les grands spécimens, à l'exception du site 3 , les pics d'abondance sont par contre notés sur les sites 1,2 et 4 selon les espèces. Ainsi, montrent-ils leur capacité de distribution plus large que les immatures. Cela pourrait s'expliquer par leur capacité d'osmorégulation remarquable comparativement aux plus jeunes comme cela a été noté chez Mugil cephalus (Cardona, 2000). Les tailles moyennes sont toujours en faveur des femelles, et ce quel que soit le site, ce qui traduirait une croissance plus importante des femelles par rapport aux mâles. Les tailles moyennes les plus faibles observées sur le site 2 chez Liza dumerili, Liza falcipinnis et Mugil bananensis seraient liées à la forte présence des spécimens sexuellement indifférenciés de taille plus petite sur ce site chez ces espèces comme nous l'avons souligné. Quoique ceci ne soit pas le cas chez Mugil curema, la taille moyenne la plus faible étant notée sur le site 3. De plus, les variations des tailles moyennes de la population des différentes espèces pourraient s'expliquer d'une part par la proportion des femelles de chaque population et d'autre part aux déplacements des populations au sein de la lagune surtout en 
saison des pluies et en période de reproduction.

\section{Conclusion}

Les six espèces de Mugilidae identifiées dans la lagune de Grand-lahou montrent une spécificité dans leur occupation spatiale. En effet, Mugil curema et Mugil bananensis sont plus abondants dans la zone de contact avec la mer que dans les zones proches du grau. Cette distribution est inversée chez Liza dumerili et Liza grandisquamis. Mugil cephalus et Liza falcipinnis ont plutôt une répartition quasiuniforme. Par ailleurs, de mi-juillet à fin septembre, cette distribution spatiale est influencée par la saison des pluies et par la reproduction. En effet, les eaux de la lagune sont dessalées, exception faite de celles de la partie océanique et du grau, fréquentées par les Mugilidae pendant cette période. C'est aussi la saison de reproduction et une migration vers les sites de la reproduction. En outre, les spécimens sexuellement indifférenciés sont majoritairement distribués dans les zones proches du grau, les individus de grandes tailles sont plutôt largement distribués au sein de la lagune.

La sex-ratio globale est en faveur des mâles chez Liza dumerili, Mugil cephalus et Liza grandisquamis. Chez Mugil bananensis, Liza falcipinnis et Mugil curema, elles sont en faveur des femelles. Quant aux différentes tailles moyennes calculées, celles des femelles restent significativement supérieures à celles des mâles, exception faite chez Liza grandisquamis.

L'échantillonnage de cette étude provient d'une pêche commerciale. Les mailles des engins de pêche utilisés n'ont pas permis la capture de toutes les gammes de tailles, notamment les alevins. Il serait donc intéressant d'approfondir ces recherches à travers une pêche expérimentale afin de mieux comprendre la distribution de tous les stades de croissance.

\section{REFERENCES}

Albaret JJ, Legendre M. 1985. Biologie et écologie des Mugilidae en lagune Ebrié (Côte d'Ivoire) intérêt potentiel pour l'aquaculture lagunaire. Rev. Hydrobiol. Trop., 18(4): 281-303.

Albaret JJ. 1994. Les poissons: Biologie et peuplements. In Environnement et Ressources Aquatiques de Côte-d'Ivoire: Les Milieux Lagunaires, Durand JR, Dufour Ph, Guiral D, Zabi SGF (eds). ORSTOM: Paris; 239-279.

Albaret JJ. 2003. Mugilidae. In Faune des Poissons d'Eaux Douces et Saumâtres de l'Afrique de l'Ouest (Tome 2), Paugy D, Lévêque C, Teugels GG (eds). IRD Editions: Paris; 601-611.

Albaret JJ, Simier M, Sambou Darboe F, Ecoutin JM, Raffray J, Tito de Morais L. 2004. Fish diversity and distribution in The Gambia estuary, West Africa in relation to environmental variables. Aquat. Living Resour., 17: 35-46.

Ameur B, Bayed A, Benazzou T. 2003. Rôle de la communication de la lagune de Merja Zerga (Garb, Maroc) avec l'océan Atlantique dans la reproduction d'une population de Mugil cephalus L. (Poisson Mugilidae). Bull. Instit. Scient. Rabat. Sect. Scien. Vie, 25: 77-82.

Bernadon M, Mohamed Vall MO. 2005. Le Mulet en Mauritanie : biologie, écologie, pêche et amenagement, p. 53.

Billard R. 1997. Les Poissons d'Eau Douce des Rivières de France. Identification, Inventaire et Répartition des 83 Espèces. Delachaux et Niestlé: Lausanne; 192.

Bruslé J. 1981. Sexuality and biology of reproduction in grey mullets. In Aquaculture of Grey Mullet, Oren $\mathrm{OH}$ 
(ed). Cambridge University Press: Cambridge; 94-154.

Bruslé J, Bruslé S. 1977. Les Muges de Tunisie: Pêche lagunaire et biologie de la reproduction de trois espèces (Mugil capito, Mugil cephalus et Mugil chelo) des lacs d'Ickeul et de tunis. Rapp. Comm. Int. Mer Méditer., 24(5): 101-130.

Cardona L. 2000. Effects of salinity on the habitat selection and growth performance of Mediterranean flathead grey mullet Mugil cephalus (Osteichthyes, Mugilidae). Estuarine, Coastal and Shelf Science, 50: 727-737.

Cardona L. 2006. Habitat selection by grey mullets (Osteichthyes: Mugilidae) in Mediterranean estuaries: the role of salinity. Scientia Marina, 70: 443-455.

Chang CW, Iizuka Y. 2012. Estuarine utilization and movement pattern of the seven sympatric Mugilidae fishes in the Tatu Creek estuary, central western Taiwan. Estuarine, Coastal and Shelf Science, 106: 121-126

Chikou A. 2006. Etude de la démographie et de l'exploitation halieutique de six espèces de poissons-chats (Teleostei, Siluriformes) dans le delta de l'Ouémé au Bénin. Thèse Doct. Univ. Liège, Belgique, p. 459.

Diouf PS. 1991. Guide de détermination rapide des mulets des estuaires sénégalais. Centre de Recherches Océanographiques de Dakar-Tiaroye ; Document Scientifique No. 129 : 1-13.

Durand R, Skubich M. 1979. Recherches sur les Lagunes Ivoiriennes. Doc. Multigr. C. R. O.: Abidjan; 55.

Durand JR, Chantraine JM. 1982. L'environnement climatique des lagunes ivoiriennes. Rev. Hygrobiol. Trop., 15(2): 85-113.

El-Zarka S, El-Sedfy HM. 1970. The biology of Mugil saliens (Risso) in Lake Qarun.
U.A.R. Bull. Inst. Ocean. Fish., Cairo, 1: $1-26$.

Fehri Bedoui R, Gharbi H, El Abed A. 2002. Période de reproduction et maturité sexuelle de Liza aurata (poisson, Mugilidae) des côtes Est et Sud tunisiennes. Bulletin de l'INSTM, 29: 1115.

Harrison IJ. 2008. Mugilidae. In Poissons d'Eaux Douces et Saumâtres de BasseGuinée, Ouest de l'Afrique Centrale (vol. 2), Stiassny MLJ, Teugels GG, Hopkins CD (eds). IRD Editions : Paris; 450-471.

Keith P, Allardi J. 2001. Atlas des Poissons d'eau douce de France. Patrimoines Naturels, 47(387).

Konan KS, Kouassi AM, Adingra AA, Bongui BK, Dago G. 2008. Variations saisonnières des paramètres abiotiques des eaux d'une lagune tropicale: La lagune de Grand-lahou, Côte d'ivoire. Europ. J. Scientif. Research, 21(3): 376393.

Koutrakis ET. 2004. Temporal occurrence and size distribution of grey mullet juveniles

(Pisces, Mugilidae) in the estuarine systems of the Strymonikos Gulf (Greece). J. Appl. Ichthyol., 20: 76-78.

Lae R. 1982. Premières observations sur la pêche en lagune de Grand-Lahou. Thèse de DEA en Océanographie Tropicale, Univ. Brest, France, p. 30.

Marin BJ, Quintero A, Bussiere D, Dodson JJ. 2003. Reproduction and recruitment of white mullet (Mugil curema) to a tropical lagoon (Margarita Island, Venezuela) as revealed by otolith microstructure. Fishery Bulletin, 101: 809-821.

Nouiri H, Berrebi P, Lbadaoui K, Benhoussa A, Jaziri H, Benazzou T. 2007. Variation saisonnière de l'abondance du recrutement et de la distribution de taille chez les juvéniles de Mugilidae de la 
lagune de Merja Zerga (littoral atlantique, Maroc). Cybium, 31(4): 427-434.

Ould Mohamed Vall M. 2004. Etude de la dynamique des systèmes d'exploitation et de l'écobiologie de la reproduction de trois Mugilidae: Mugil cephalus (Linnaeus, 1758), Liza aurata (Perugia, 1892) et Mugil capurrii (Risso, 1810), analyse de leurs stratégies d'occupations des secteurs littoraux mauritaniens et de leurs possibilités d'aménagement. Thèse Doct., Univ. Nice-Sophia Antipolis, France, p. 146.

Perez-Ruzafa A, Quispe-Becerra JI, Garcia-Charton JA, Marcos C. 2004. Composition, structure and distribution of the ichthyoplankton in a Mediterranean coastal lagoon. J. Fish Biol., 64: 202-218.
Pombo L, Elliott M, Rebelo JE. 2005. Environmental influences on fish assemblage distribution on an estuarine coastal lagoon, Ria de Aveiro (Portugal). Sci. Mar., 69: 143-159.

Soyinka OO, Kuton MP, Ayo-Olalusi CI. 2010. Seasonal distribution and richness of species in the Badagry Lagoon, South West, Nigeria. Estonian J. Ecol., 59: 147157.

Trape S, Durand JD, Guilhaumon F, Vigliola L, Panfili J. 2009. Recruitment patterns of young-of-the-year mugilid fishes in a West African estuary impacted by climate change. Estuarine Coastal and Shelf Science, 85: 357-367. 